\title{
Postural Orthostatic Tachycardia Syndrome unmasked by successful treatment of primary aldosteronism
}

Abbi Lulsegged, Nataliya Zuyeva, Nicholas Gall

Kings College Hospital NHS Foundation Trust, London, United Kingdom

\section{King's College Hospital W/HS}

NHS Foundation Trust

\section{History:}

\section{0-year-old woman}

History of chronic, symptomatic hypokalaemia and hypertension.

On examination weight $69.9 \mathrm{~kg}$, height 1.65 meters, BP $163 / 99$, pulse 70 ,

Heart sounds normal and examination of the abdomen was unremarkable. ECG was normal.

Despite taking potassium supplements, repeat blood tests showed Sodium $148 \mathrm{mmol} / \mathrm{L}$, potassium

$3.3 \mathrm{mmol} / \mathrm{L}$, Magnesium 0.87, Creatinine 56, eGFR > 90. Spot Urine potassium $25 \mathrm{mmol} / \mathrm{L}$.

Primary aldosteronism was suspected

Further tests were arranged.

Renin was undetectable $(<1.1 \mathrm{ng} / \mathrm{L})$

Serum aldosterone $772 \mathrm{pmol} / \mathrm{L}$.

Saline suppression test showed non-suppressible aldosterone levels.

Glucocorticoid-remediable aldosteronism was excluded. MRI failed to show a adrenal lesion.

Adrenal vein sampling confirmed a unilateral, right sided adrenal source for the aldosterone excess

Underwent successful right adrenalectomy.

Histology was supportive of the diagnosis

Shortly after the operation she started to experience significant postural dizziness.

Relative suppression of aldosterone secretion by the contralateral gland suspected.

Fludrocortisone was started after a short synacthen test excluded adrenal deficiency.

She required increasing doses of fludrocortisone but despite this remained symptomatic.

Additionally she experienced headaches, fatigue, cold intolerance and breathlessness.

Therefore a neurocardiogenic process was suspected and she was referred to Cardiology.

Tilt table test showed her heart rate gradually rose to $138 \mathrm{bpm}$.

This was thought to be consistent with a POTs type response.

Characterised by autonomic instability resulting in orthostatic intolerance.

Increase in Heart Rate $>30 \mathrm{bpm}$ or rate $>120 \mathrm{bpm}$ after standing $(5-30 \mathrm{mins})$.

Impaired venous innervation, alpha-1-adrehergic receptor denervation/insensitivity and/or beta-

adrenergic receptor hypersensitivity contribute to reduce venous return on standing.

Associated symptoms include fatigue, sweating, palpitations, headaches, postprandial hypotension.

Typically affects women (5:1) aged $15-50$ years.

Conclusions

Thus our patient had confirmed primary aldosteronism due to a adrenal adenoma, removal of which helped to unmask POTS.

We hypothesise that the excess mineralocorticoid activity from the adenoma hyper secreting aldosterone helped to mask the neurocardiogenic process. 